\title{
Polarization Properties of Specular and Dense Multipath Components in a Large Industrial Hall
}

\author{
Davy P. Gaillot ${ }^{1}$, Emmeric Tanghe ${ }^{2}$, Wout Joseph ${ }^{2}$, Pierre Laly ${ }^{1}$, Brecht Hanssens ${ }^{2}$, Martine Liénard ${ }^{1}$, and Luc \\ Martens $^{2}$
}

${ }^{1}$ University of Lille 1, Institute of Electronics, Microelectronics, and Nanotechnology, Building P3, F-59655 Villeneuve d'Ascq, France, e-mail: davy.gaillot@univ-lille1.fr

${ }^{2}$ Ghent University/iMinds, Department of Information Technology, Gaston Crommenlaan 8 box 201, B-9050 Ghent, Belgium, e-mail: emmeric.tanghe@intec.ugent.be

\begin{abstract}
This paper presents an analysis of the polarization characteristics of specular and dense multipath components (SMC \& DMC) in a large industrial hall based on frequency-domain channel sounding experiments at $1.3 \mathrm{GHz}$ with 22 $\mathrm{MHz}$ bandwidth. The RiMAX maximum-likelihood estimator is used to extract the full polarimetric SMC and DMC from the measurement data by taking into account the polarimetric radiating patterns of the dual-polarized antennas. Cross-polar discrimination (XPD) values are presented for the measured channels and for the SMC and DMC separately.

\section{Introduction}

Over the course of the last decade, the physical view of the radio channel has undergone an important change. Before that, the radio channel was commonly considered to be but a collection of SMC that have well-defined discrete locations in the different radio channel dimensions (e.g., space, frequency, time, etc.). Nowadays, it is widely accepted that part of the radio channel is also continuous across these dimensions. This part is put under the umbrella of DMC [1]. Among other sources, these dense multipath components originate from distributed diffuse scattering on electrically small objects. Naturally, the introduction of DMC in the physical model of the radio channel means that common radio channel parameters have to be re-evaluated for dense multipath as well. This contribution fits within this effort. In this paper, we present an analysis of the SMC and DMC cross-polarization discrimination (XPD) parameters in a large industrial environment. The analysis is based on frequency-domain channel sounding experiments at $1.3 \mathrm{GHz}$ with 22 $\mathrm{MHz}$ bandwidth. The RiMAX maximum-likelihood estimator is used to extract the full polarimetric SMC and DMC from the channel sounding data [1].
\end{abstract}

\section{Methodology}

\subsection{Measurement environment and procedure}

The propagation environment under consideration is a large industrial hall located in Zwijnaarde, Belgium. The hall has dimensions $21.3 \times 77.2 \times 12.2 \mathrm{~m}^{3}$ and is dedicated to the research of concrete technology. The main inventory consists of large metallic machinery used for, i.a., testing the robustness of concrete structures. The dominant building material for walls, floor, and ceiling is concrete. Frequency-domain channel sounding measurements were performed in the industrial hall. A vector network analyzer was used to probe the radio channel in a $22 \mathrm{MHz}$ bandwidth centered around $1.3 \mathrm{GHz}$. In this frequency band, 1601 uniformly spaced frequency points were sampled. At both link ends, a virtual antenna array was created by an antenna mounted on an automated rotating arm. At both transmit and receive side, the virtual array was a planar horizontal uniform circular array with radius $15 \mathrm{~cm}$ and consisting of 12 antenna elements. As transmitting and receiving antenna, dual-polarized rectangular patch antennas were used. Both antennas were $1.60 \mathrm{~m}$ above ground level during measurements. In total, $29 \mathrm{Tx}-\mathrm{Rx}$ links were measured under either strong LineOf-Sight (LOS) or Obstructed LOS (OLOS) conditions.

\subsection{Specular and dense multipath estimation}

Typically, the full polarimetric measured channel matrix $\boldsymbol{H}$ is given by:

$$
\boldsymbol{H}=\left[\begin{array}{ll}
\boldsymbol{h}_{H H} & \boldsymbol{h}_{H V} \\
\boldsymbol{h}_{V H} & \boldsymbol{h}_{V V}
\end{array}\right]
$$

The sampled array response vector $\boldsymbol{h}_{X Y} \in \mathbb{C}^{M_{R} M_{T} M_{F} \times 1}$ can be written as the sum of an SMC part $\boldsymbol{s}_{X Y}$ and a DMC part $\boldsymbol{d}_{\boldsymbol{X}}$. The subscripts $X$ and $Y$ denote the polarization of the receiving and the transmitting antenna, respectively: $X$ and $Y$ are either horizontal $(H)$ or vertical $(V)$. It is commonly assumed that $\boldsymbol{h}_{X \boldsymbol{Y}}$ follows a multivariate circular symmetric complex Gaussian process [1]: 


$$
\boldsymbol{h}_{X Y}=\boldsymbol{s}_{X Y}\left(\boldsymbol{\theta}_{s, X Y}\right)+\boldsymbol{d}_{X Y}\left(\boldsymbol{\theta}_{\boldsymbol{d}, X Y}\right) \text { and } \boldsymbol{h}_{X Y} \sim \mathcal{N}_{C}\left(\boldsymbol{s}_{X Y}\left(\boldsymbol{\theta}_{s . X Y}\right), \boldsymbol{R}_{X Y}\left(\boldsymbol{\theta}_{\boldsymbol{d}, X Y}\right)\right)
$$

The parameter vector $\boldsymbol{\theta}_{\boldsymbol{s}, \boldsymbol{X} \boldsymbol{Y}}$ groups the parameters associated with the SMCs. On one hand, these include the geometrical parameters that locate the specular path in the propagation environment: the angles of arrival and departure and the time-delay of arrival. The geometrical SMC parameters are identical across all four polarization subchannels $X Y$. On the other hand, $\boldsymbol{\theta}_{\boldsymbol{s}, \boldsymbol{X Y}}$ also includes the complex amplitude $\gamma_{X Y}$ of the SMCs. The complex amplitude will differ between different polarization subchannels because of the polarization-dependency of electromagnetic interactions (reflections, diffractions, etc.).

The DMC power delay profile $\psi_{X Y}(\tau)$ as a function of time delay $\tau$ is described by an exponential decay:

$$
\psi_{X Y}(\tau)=\alpha_{1, X Y} e^{-B_{d, X Y}\left(\tau-\tau_{d, X Y}\right)}+\alpha_{0, X Y}
$$

In (3), $\alpha_{1, X Y}, B_{d, X Y}, \tau_{d, X Y}$, and $\alpha_{0, X Y}$ are four parameters which fully describe the DMC of polarization subchannel $X Y$ and are gathered into the DMC parameter vector $\boldsymbol{\theta}_{\boldsymbol{d}, \boldsymbol{X Y}}$. Maximum-likelihood estimators of $\boldsymbol{\theta}_{\boldsymbol{s}, \boldsymbol{X Y}}$ and $\boldsymbol{\theta}_{\boldsymbol{d}, \boldsymbol{X Y}}$ are obtained from the channel sounding data using the iterative RiMAX multipath estimation algorithm [1]. The de-embedding of the full polarimetric complex radiation patterns of the measurement antennas is based on the concept of the Effective Aperture Distribution Function (EADF) detailed in [2].

\section{Results}

\subsection{Specular and dense multipath power}

In this section, the total measured power $\mathrm{P}_{\mathrm{T}}$ as well as the estimated SMC and DMC power $\left(\mathrm{P}_{\mathrm{SMC}} \& \mathrm{P}_{\mathrm{DMC}}\right)$ are investigated as a function of shadowing situation and $\mathrm{Tx}-\mathrm{Rx}$ distance. Small-scale fading was removed by averaging over the $12 \times 12=144$ possible combinations of $\mathrm{Tx}$ and $\mathrm{Rx}$ antenna array elements and the $4 \mathrm{Tx}-\mathrm{Rx}$ polarization combinations. Fig. 1 presents power versus distance for LOS (left) and OLOS (right). The results indicate that the power is decreasing with distance as expected but also that it fades more rapidly for OLOS scenarios. The contribution of the SMC to the total power is larger for LOS than OLOS due to the high contribution of the LOS component. Furthermore, the SMC and DMC power show a strong correlation of 90\% (LOS) and 95\% (OLOS). This can be explained by the fact that the DMC can be interpreted as the non-coherent superposition of paths with weaker SNRs which still follow the same propagation mechanisms as the SMC. The DMC to total power ratio $\mathrm{P}_{\mathrm{DMC}} / \mathrm{P}_{\mathrm{T}}$ for LOS (OLOS) has a mean value of $14.3 \%(40.5 \%)$ and standard deviation of $7 \%(10 \%)$ in agreement with recently reported values for indoor [3] and industrial environments [4].
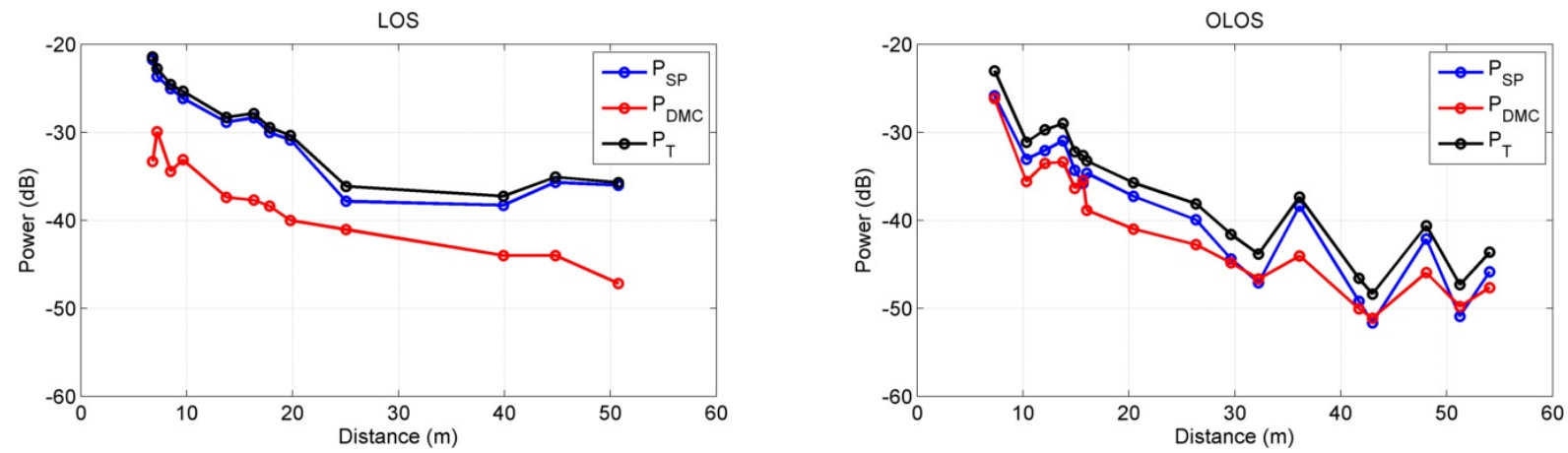

Fig. 1: $P_{\mathrm{T}}, P_{\mathrm{SP}}$, and $P_{\mathrm{DMC}}$ (in $\mathrm{dB}$ ) as a function of distance (in $\mathrm{m}$ ) for LOS (left) and OLOS (right) scenarios.

\subsection{XPD of measured channels}

In this section, the XPD values are studied from the measured polarimetric channels $\boldsymbol{H}$. The small-scale variations are removed by averaging over the 144 combinations of Tx and Rx antenna array elements. The XPD for $\mathrm{H}$ and V for each position are given by:

$$
\begin{aligned}
& X P D_{H}(\mathrm{~dB})=10 \log _{10}\left\langle\frac{\left|h_{H H}\right|^{2}}{\left|h_{H V}\right|^{2}}\right\rangle \\
& X P D_{V}(\mathrm{~dB})=10 \log _{10}\left\langle\frac{\left|h_{V V}\right|^{2}}{\left|h_{V H}\right|^{2}}\right\rangle
\end{aligned}
$$


Table I presents the $\mathrm{XPD}_{\mathrm{H} / \mathrm{V}}$ values averaged over the LOS and OLOS links separately. In spite of the fact that the reported XPD values are close to those found in the literature for indoor environments [5-7], it is noted that there is slightly more depolarization for $\mathrm{V}$ than for $\mathrm{H}$.

TABLE I

Measured XPD values (in dB)

\begin{tabular}{|c|c|c|}
\cline { 2 - 3 } \multicolumn{1}{c|}{} & $\left\langle\mathrm{XPD}_{\mathrm{H}}\right\rangle$ & $\left\langle\mathrm{XPD}_{\mathrm{V}}\right\rangle$ \\
\hline LOS & 12.2 & 11.05 \\
\hline OLOS & 8.48 & 7.58 \\
\hline
\end{tabular}

\subsection{XPD of specular and dense multipath}

In this section, the XPD values are calculated for the SMC and DMC separately. Per Tx-Rx link, XPD $\mathrm{H} / \mathrm{v}$ is calculated for each individual specular path based on the estimated complex amplitudes $\gamma_{X Y}$ of that path. It is worth to mention that RiMAX estimated on average around 200 specular paths per Tx-Rx link. For the DMC, XPD $\mathrm{H} / \mathrm{v}$ is calculated based on the total DMC power for each of the four H/V combination (i.e., the integral of (3)).

Fig. 2 presents the statistics of $\mathrm{XPD}_{\mathrm{H}}$ and $\mathrm{XPD}_{\mathrm{V}}$ as a function of distance for both LOS and OLOS scenarios. The LOS component has been removed to avoid any strong bias as this component does not suffer from depolarization mechanisms. Boxplots were used to assess the XPD distribution of the SMC. For comparison, the averaged XPD of the measured channel and the DMC are also shown. From Fig. 2, it is observed that SMC, DMC, and measured XPD do not correlate heavily with distance.
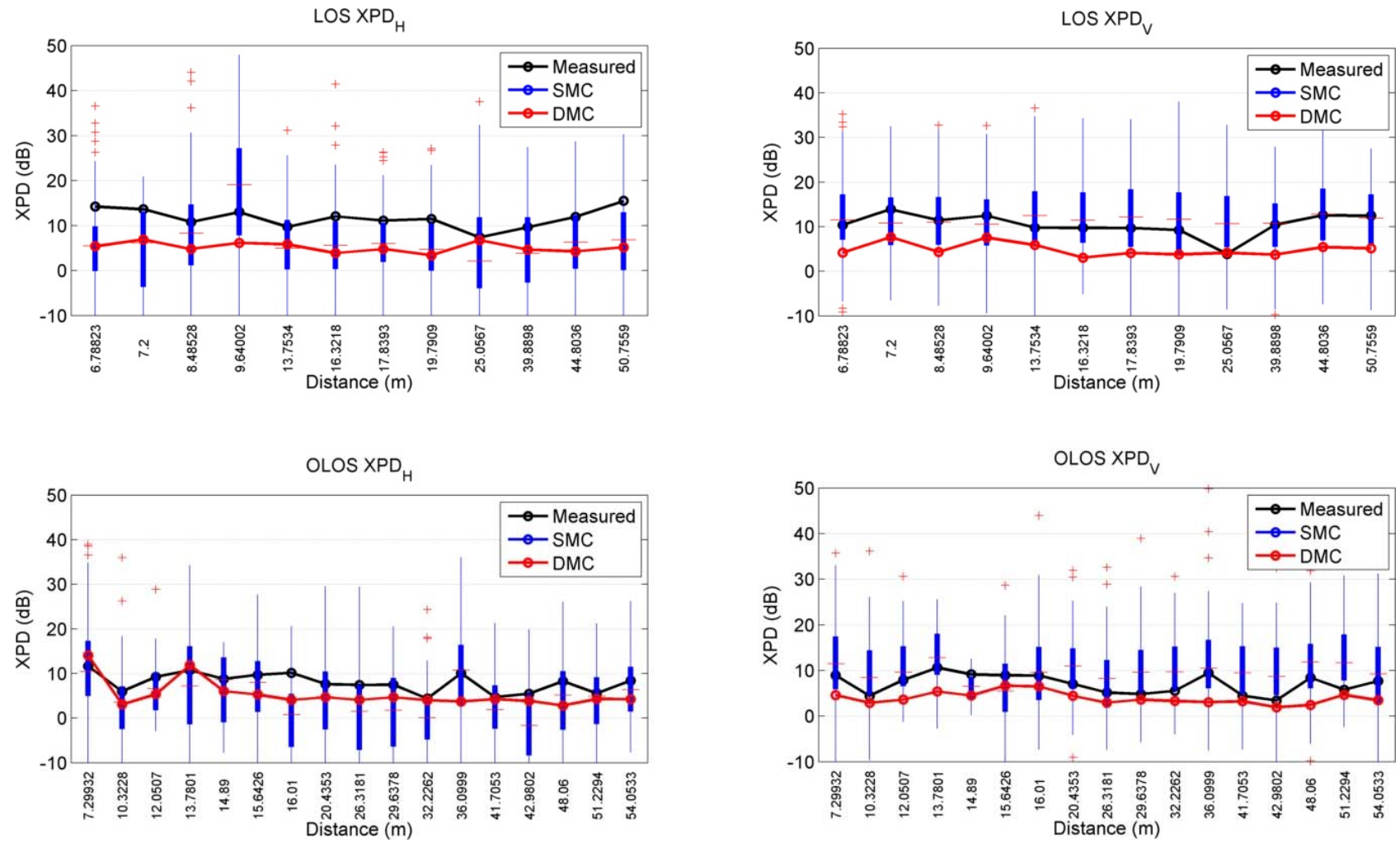

Fig. 2: SMC, DMC, and measured XPD values (in $\mathrm{dB}$ ) as a function of distance (in m) for $\mathrm{H}$ (left column) and V (right column) and for LOS (top row) and OLOS (bottom row) scenarios.

Table II summarizes the XPD values averaged over distance. For the SMC, the median value (.) 0.5 of the path XPDs per location is averaged. For the SMC, the power imbalance between $<\left(\mathrm{XPD}_{\mathrm{H}}\right)_{0.5}>$ and $<\left(\mathrm{XPD}_{\mathrm{V}}\right)_{0.5}>$ contrasts with the averaged XPD computed directly from the measured channels (Table I). The results demonstrate that the propagation characteristics are rather different between $\mathrm{V}$ and $\mathrm{H}$ polarized specular paths. This could be attributed to the Brewster angle effect for $\mathrm{H}$ polarized transmission [8-9]; this effect could be potentially important in an environment where a large number of machines and metallic/concrete scatterers can be found in the azimuthal plane of the antennas. It is known that the $\mathrm{V}$ depolarization is strongly dependent on the elevation angle. Reflections off the high ceiling will most likely have high elevation angles, giving rise to smaller XPDs. However, these reflections will likely be heavily attenuated because of their large path length. Instead, the Rx will most likely receive high SNR signals from single-bounce scattering off the floor and scatterers spread out between Tx and Rx since both arrays are at the same height. These V polarized paths are 
not expected to suffer much from depolarization due to their low elevation angles. The prevalence of low elevation angles is confirmed by the fact that $90 \%$ of the estimated paths arrive with an elevation angle smaller than $50^{\circ}\left(20^{\circ}\right.$ at $70 \%)$.

The average XPD values for the DMC in Table II are larger than the $0 \mathrm{~dB}$ value recently reported in indoor environments [7]. This shows that full DMC depolarization is not achieved in this large volume environment. Again, this may be attributed to the position asymmetry of the arrays when measuring the environment due to the high ceiling. It appears that DMC depolarization is almost constant across H/V and LOS/OLOS with a mean value of $4.45 \mathrm{~dB}$ and standard deviation less than $3 \mathrm{~dB}$ for distances greater than $15 \mathrm{~m}$. The SMC and DMC XPD values for $\mathrm{H}$ are very close highlighting the fact that they are both affected by the same propagation effects. Conversely, a larger depolarization is observed for V polarized DMC compared to V polarized SMC. Hence, the depolarization for V channels is strongly provided by DMC propagation mechanisms.

TABLE II

Averaged Median XPD for SMC and Averaged XPD for DMC (in dB)

\begin{tabular}{|c|c|c|c|c|}
\cline { 2 - 5 } \multicolumn{1}{c|}{} & \multicolumn{2}{c|}{ SMC } & \multicolumn{2}{c|}{ DMC } \\
\hline & $\left\langle\left(\mathrm{XPD}_{\mathrm{H}}\right)_{0.5}>\right.$ & $\left.<\left(\mathrm{XPD}_{\mathrm{V}}\right)_{0.5}\right\rangle$ & $\left\langle\mathrm{XPD}_{\mathrm{H}}\right\rangle$ & $\left\langle\mathrm{XPD}_{\mathrm{V}}\right\rangle$ \\
\hline LOS & 5.72 & 11.53 & 5.29 & 5.17 \\
\hline OLOS & 6.11 & 9.98 & 6.84 & 4.16 \\
\hline
\end{tabular}

\section{Conclusion}

In conclusion, the polarization characteristics of SMC \& DMC have been investigated in a large industrial hall, based on frequency-domain channel sounding experiments at $1.3 \mathrm{GHz}$ with $22 \mathrm{MHz}$ bandwidth. 29 positions were measured under either strong LOS or OLOS scenarios. The full-polarimetric SMC and DMC were estimated with RiMAX by de-embedding the polarimetric radiating patterns of the dual-polarized antennas. Measured XPD values were shown to be in agreement with smaller volume indoor environments. Strong de-embedded SMC depolarization is observed for $\mathrm{H}$ in OLOS scenarios. On the other hand, DMC depolarization is observed to be rather strong compared to previous studies due to the high ceiling but uniform across all scenarios, polarization, and distances. The results also show fractional DMC power of $15 \%$ for LOS and $40 \%$ for OLOS with no significant monotonic relationship with the transmitter-receiver distance and correlation between each other. Finally, a mean reverberation time of 70 ns with low standard deviation validates the room electromagnetics theory for transmitter - receiver distances larger than $15 \mathrm{~m}$ with no significant difference between polarized subchannels.

\section{References}

[1] A. Richter, "Estimation of Radio Channel Parameters: Models and Algorithms," Ph.D. dissertation, Technische Universität Ilmenau, Fakultät für Elektrotechnik und Informationstechnik, Ilmenau, DE, 2005.

[2] M. Landmann, "Limitations of Experimental Channel Characterisation,” Ph.D. dissertation, Technische Universität Ilmenau, Fakultät für Elektrotechnik und Informationstechnik, Ilmenau, DE, 2008.

[3] F. Quitin, C. Oestges, F. Horlin, and P. De Doncker, "Diffuse Multipath Component Characterization for Indoor MIMO Channels,” in European Conference on Antennas and Propagation, Barcelona, ES, April 2010, pp. 1-5.

[4] E. Tanghe, D. P. Gaillot, M. Lienard, L. Martens, and W. Joseph,”Experimental Analysis of Dense Multipath Components in an Industrial Environment," $7^{\text {th }}$ MCM COST IC1004, Ilmenau, DE, May 2013, TD(13)07015.

[5] V. R. Anreddy, M.-A. Ingram, "Capacity of measured ricean and rayleigh indoor MIMO channels at $2.4 \mathrm{GHz}$ with polarization and spatial diversity," Wireless Communications and Networking Conference, 2006. WCNC 2006. IEEE , vol.2, no., pp.946,951, 3-6 April 2006.

[6] J. M. Molina-Garcia-Pardo, J.-V.Rodríguez, L.Juan-Llacer, "Polarized Indoor MIMO Channel Measurements at 2.45 GHz," Antennas and Propagation, IEEE Transactions on , vol.56, no.12, pp.3818,3828, Dec. 2008.

[7] E. M. Vitucci, F. Mani, C. Oestges, V. Degli-Esposti, "Analysis and modeling of the polarization characteristics of diffuse scattering in indoor and outdoor radio propagation," Applied Electromagnetics and Communications (ICECom), 2013 21st International Conference on , vol., no., pp.1,5, 14-16 Oct. 2013.

[8] P. Kyritsi and D. C. Cox, "Propagation characteristics of horizontally and vertically polarized electric fields in an indoor environment: simple model and results," IEEE Vehicular Technology Conference, vol. 3, pp. 1422-1426, Oct. 2001.

[9] Y. Lostanlen and T. Tenoux, "Electromagnetic Wave Depolarization Behaviour at Different Frequencies for Various Dielectric Materials," Antennas and Propagation, 2007. EuCAP 2007. The Second European Conference on , vol., no., pp.1,6, 11-16 Nov. 2007. 